Journal of

Dentistry and Oral Health

\title{
Amniotic Membrane as Barrier Membrane in Endo-Perio Lesion - A Interdisci- plinary Approach
}

\section{Ramnath Elangovan*, Ramakrishnan Theyagarajan, Mejalla Muthiah Amala Dhas}

Adhiparasakthi Dental College and Hospital, India

*Corresponding author: Ramnath Elangovan, Adhiparasakthi Dental College and Hospital, India, Tel: 07449197007; E-mail: ramubds2@gmail.com

Received Date: October 19, 2019 Accepted Date: November 25, 2019 Published Date: November 27, 2019

Citation: Ramnath Elangovant (2019) Amniotic Membrane as Barrier Membrane in Endo-Perio Lesion - A Interdisciplinary Approach. J Dent Oral Health 6: 1-7.

\begin{abstract}
Endo- Perio lesions are the most encountered complicated condition which is difficult to diagnose and treat. The challenge in treating an Endo-Perio lesion is rectifying the Endodontic infection and regeneration of the lost alveolar bone. There are many systematic approaches and materials that are available for the treatment of the Endo-Perio lesion. Membranes used in Guided Tissue Regeneration procedures should enhance the growth of lost periodontal structures and also adapt to the surface contours of the bone and root surfaces. Amniotic membrane has the ability to adapt to surfaces and acts as a reservoir of Stem Cells which enhances the healing process. This case report presents the successful treatment of a hopelessly diseased Endo-Perio lesion using Amniotic membrane as a barrier membrane in Guided Tissue Regeneration.

Keywords: Endo-Perio lesion; Guided Tissue Regeneration; Amniotic Membrane
\end{abstract}




\section{Introduction}

The relationship between pulp and periodontium has been studied extensively regarding the diagnosis, treatment, and prognosis of the Endo -Perio lesions. Pulpal infection might proceed from the apical region through the periodontal ligament space to the marginal gingiva and give an appearance of periodontal tissue destruction, termed retrograde periodontitis [9]. The amount of bone destruction is based on the severity of the disease. Regenerative medicine is a field that is based on the concept of transplanting exogenous or stimulating endogenous stem cells to generate biological substitutes. Amnion derived cells have been reported to have the multipotent differential ability. This has attracted to become a source of stem cells for regenerative therapy [1]. In 1913, Salbella presented the first clinical report of the successful use of an amniotic membrane in the treatment of skin ulcerations. Amniotic Membrane is used in dentistry for various purposes. It is been used in treating root coverage procedures along with coronally advanced flap technique and double papilla graft $[2,3,4,5,6]$. It is also used in treating furcation defects as a barrier membrane in the regeneration of the intra bony pockets $[7,8]$. Hence The amniotic membrane serves as a better source for stem cells and may serve as an excellent barrier in Guided Tissue Regeneration procedures. The present case report describes the role of amniotic membrane as a barrier membrane in the salvation of a tooth with Endo - Perio lesion which had a questionable prognosis.

\section{Structure of Amniotic Membrane [3]}

Amniotic membranes develop from extra-embryonic tissue and consist of a material component (the deciduas) and foetal component (the chorionic plate). Chorionic villiholds these two parts and forms a connection between the cytotrophoblastic shell of the chorionic sac and the decidua basalis. The amniochorionic membrane forms the outer limits of the sac that encloses the fetus, while the innermost layer of the sac is the Amniotic Membrane. Its thickness is about 10-15 micrometers and made up of two foetal membranes; the inner layer is the amniotic membrane and the outer layer is chorion. There are two types of cells in the Amniotic Membrane with embryological origins differing from one another: amnion epithelial cells derived from embryonic ectoderm and amnion mesenchymal cells from embryonic mesoderm.

The ultrastructure of Amniotic Membranereveals to be a transparent, thin, avascular composite membrane composed of three major layers: an epithelial layer, a thick basement membrane, and mesenchyme which is avascular comprising of colla- gen. Amniotic Membrane has no blood vessels or nerves; instead, the nutrients which are required are supplied by diffusion out of the amniotic fluid and/or from the underlining deciduadirectly. The amniotic epithelial cell layer consists of a single layer of flat, cuboidal and columnar cells that are in direct contact with the amniotic fluid. It is from this layer that Amniotic Multi Potent Stem Cells (AMSC) are isolated and stored to be used for regenerating tissues. Macrophages and fibroblast-like mesenchymal cells are present in the amniotic mesoderm layer. The appearance of the basement membrane of the amnion is just like the basement membrane found in the gingiva, conjunctiva and other parts of the body.

\section{Types of Amniotic Membrane [1]}

\section{Frozen membrane}

Freezing of the Amniotic membrane is done by passing through liquid nitrogen at $-196^{\circ} \mathrm{F}$. The Cooling process makes the membrane void of microorganisms, immunologically inert material without antigenicity and preserves the membrane for an indefinite time, Cryopreservation with dimethyl sulphoxide (DMSO) at $-80^{\circ} \mathrm{C}$ allows retention cells in the Amniotic Membrane at approximately 50\% for several months. The several angiogenic growth factors and cytokines are removed during cryopreservation of the Amniotic Membrane. It has been noted that the storage of the Amniotic Membrane in glycerol at $4^{\circ} \mathrm{C}$ results in immediate cell death.

\section{Freeze-dried irradiated (lyophilized)}

After obtaining the Amniotic membrane from the placenta, it is freeze-dried at $-60^{\circ} \mathrm{C}$ under vacuum (atmospheric pressure 102) for 48 hours. Then it is irradiated with 2.5 mega rads ( $25 \mathrm{~K}$ Gray) inside a batch type cobalt-60 irradiator. By the method of freeze-drying, there is sublimation of liquid moisture of membrane to the gaseous state without having undergone the intermediate solid stage. By this method, the membrane maintains its original size and shape by minimizing cell rupture. The freeze-dried membrane can be used immediately after soaking it in normal saline for 1 minute.

\section{Properties of Amniotic Membrane}

The Multi Potent Stem Cells (MPSC) present in the Amniotic Membrane have the ability to accelerate the inflammatory phase towards the proliferative phase which is critical for treating chronic wounds like periodontitis[4]. The properties of the amniotic membrane that accelerates wound healing are:

- Immunomodulative and Immune Privilege

JScholar Publishers

J Dent Oral Health 2019 | Vol 6: 401 
- Anti-Microbial (broad-spectrum against bacteria, fungi, protozoa, and viruses)

- Reduction of pain

- Anti-Scarring and Anti-inflammatory effects

- Tissue repair with enhanced bone remodeling, osteogenesis, and chondrogenesis.

- Accelerating fibrogenesis and angiogenesis

- Increased extracellular matrix deposition

- Potent source of mesenchymal stem cells

\section{Case Report}

A 42 years old male patient reported with the chief complaint of pain and swelling in the upper front tooth region for the past week and wanted to be treated. The patient was referred to the Department of Periodontology for management. On intraoral examination, a swelling measuring roughly about $6 \times 6 \mathrm{~mm}$ along with pus discharge in relation to 23 was seen (Figure:1.A). The tooth was tender on percussion and exhibited Grade III mobility. The radiographic examination showed a vertical bone loss in both the mesial and distal aspects of 23(Figure: 2). The condition was diagnosed as an Endo-Perio lesion with the Primary Periodontal origin of the infection. The patient's medical and dental histories were non-contributory. The patient was not willing for extraction. Hence, Emergency Phase treatment was carried out by incision and drainage (Figure:1.B) followed by Phase I therapy of Scaling and Root Planing. The patient was put under antibiotic therapy of Amoxycillin 500mg and Metronidazole 400mg thrice daily for five days and NSAID pain killer Aceclofenac 100mg twice daily for three days.

After 7 days, the Intraoral examination showed a complete reduction of swelling and probing the depth of about $8 \mathrm{~mm}$ (Figure: 3). The clinical attachment level was $11 \mathrm{~mm}$. The tooth (23) was tested for vitality with Electric Pulp tester (EPT) which showed no response indicating the tooth was non-vital. Root canal procedure was planned, Access Opening along with Biomechanical Preparation was done and Calcium Hydroxide intracanal medication was placed during the second visit. On the third visit, mobility got reduced hence occlusal reduction was done rather than splinting. Obturation was done short of the apex in 23 since the canal was calcified and could not be negotiated further (Figure: 4). Localized flap Surgery was planned in relation to 23.
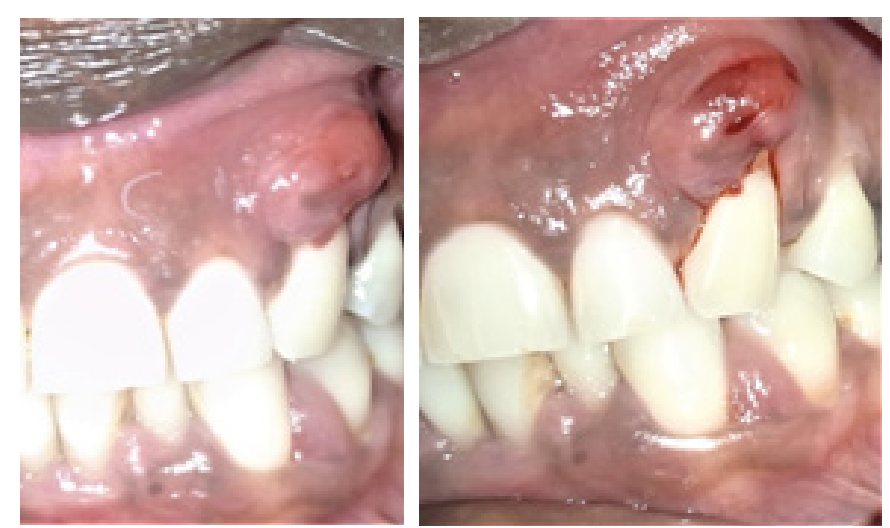

Figure 1: A). Preoperative image showing swelling and pus discharge in relation to $23, \mathrm{~B})$. Incision and drainage done.

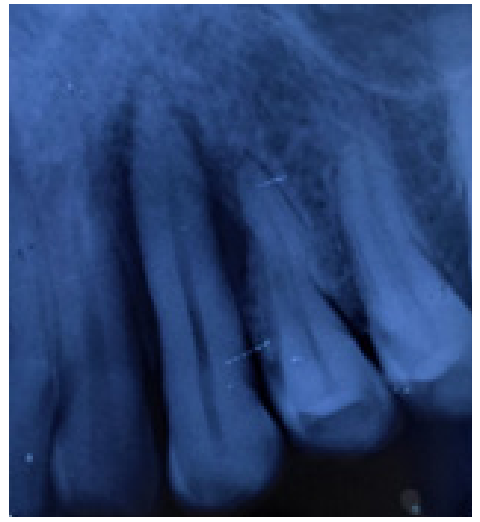

Figure 2: Preoperative Radiograph

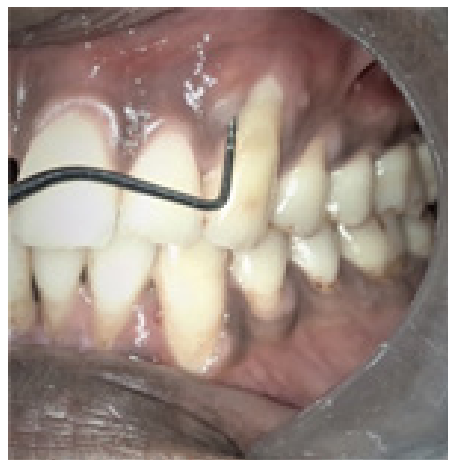

Figure 3: 1-week post-operative image showing a probing depth of $8 \mathrm{~mm}$



Figure 4: Root canal performed in consecutive visits 


\section{Surgical Procedure}

The surgical site was properly isolated and anesthetized with $2 \%$ lignocaine hydrochloride (1:2,00,000 adrenaline) manufacture details. Crevicular, interdental incisions were given from the distal aspect of 21 to the mesial aspect of 25 and two vertical incisions were placed in the distal line angle of 21 and in a distal line angle of 24. A full-thickness mucoperiosteal flap was reflected with wider base (Figure: 5) where dehiscence of the labial cortical plate was found, de-granulation was done, irrigated with normal saline, osseous defect was filled with Osseograft (Xenograft) (Figure: 6) and Amniotic membrane (Freeze -Dried, Irradiated Amnion) obtained from TATA MEMORIAL HOSPITAL TISSUE BANK - Mumbai was placed over the osseous defect (Figure:7\&8). The flap was approximated and secured with interrupted sutures using 3-0 silk suture material (Figure: 9). Coe-Pak periodontal dressing was given over the surgical site (Figure: 10).

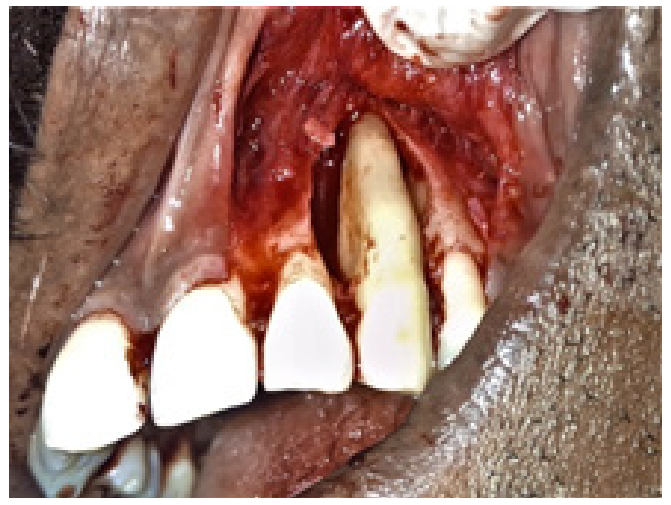

Figure 5: Full-thickness mucoperiosteal flap raised showing the osseous defect

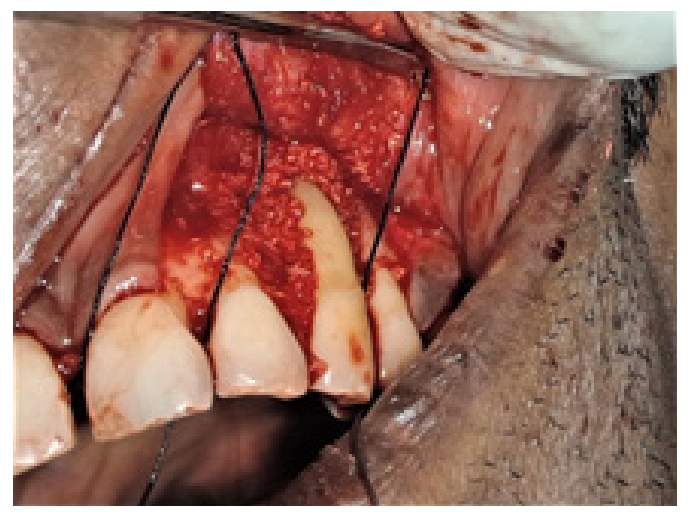

FIGURE 6: Osseous defect filled with the bone graft (allograft)

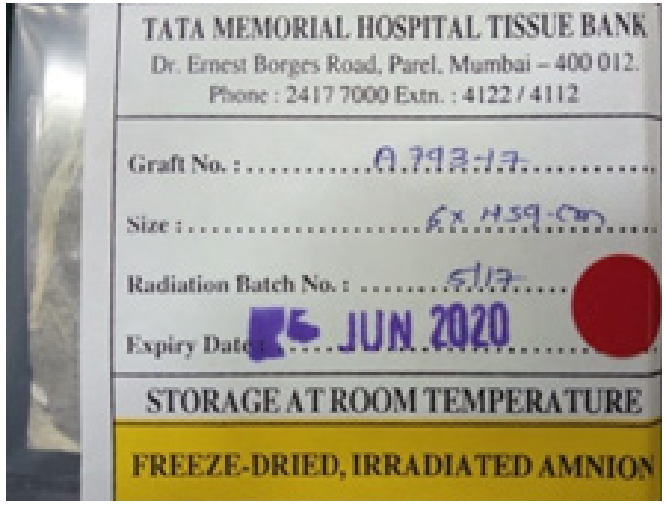

Figure 7: Freeze - Dried, Irradiated Amnion Membrane

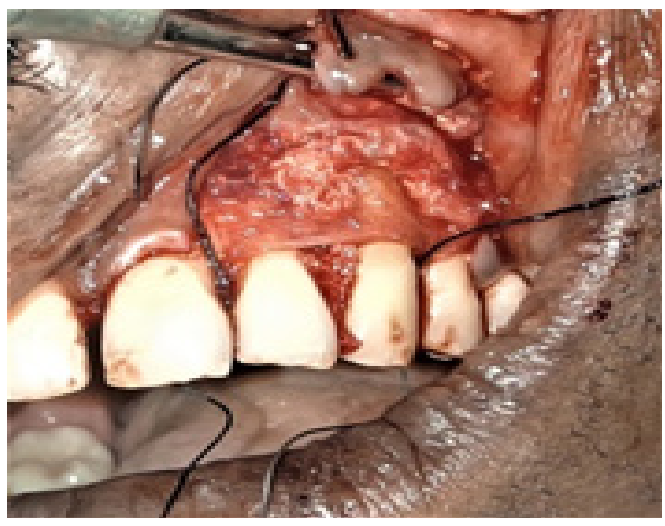

Figure 8: Amniotic membrane placed over the bone graft in osseous defect

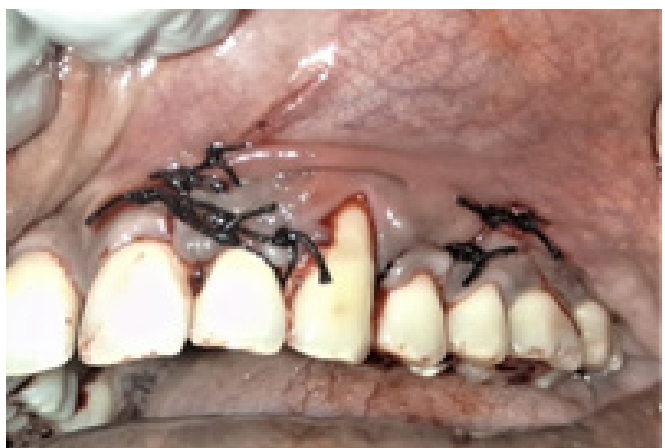

Figure 9: Flap approximated with interrupted sutures

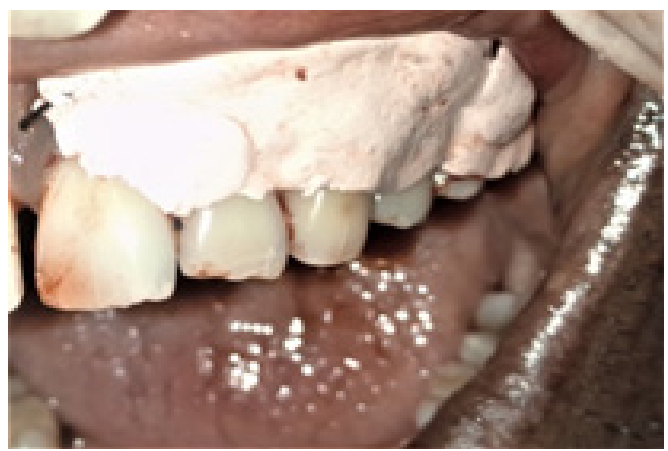

Figure 10: Coe-Pak dressing placed over the surgical site 


\section{Postoperative Instructions}

Analgesics were prescribed twice daily for three days for postoperative pain. $0.2 \%$ chlorhexidine digluconate was also prescribed to be used twice daily for 3 weeks. The patient was asked not to brush at the surgical site for 2 weeks.

\section{Result}

After 7 days of surgery, periodontal dressing and sutures were removed, healing was satisfactory (Figure: 11). The patient was again assessed for healing at one month (Figure: 12) and 6 months interval (Figure: 13). The Intraoral Periapical Radiograph at the time period of 6 months showed excellent bone formation (Figure: 15), there was a reduction in pocket depth to $3 \mathrm{~mm}$ (Figure: 14) and the Clinical At tachment level was $6 \mathrm{~mm}$. The tooth became clinically firm.

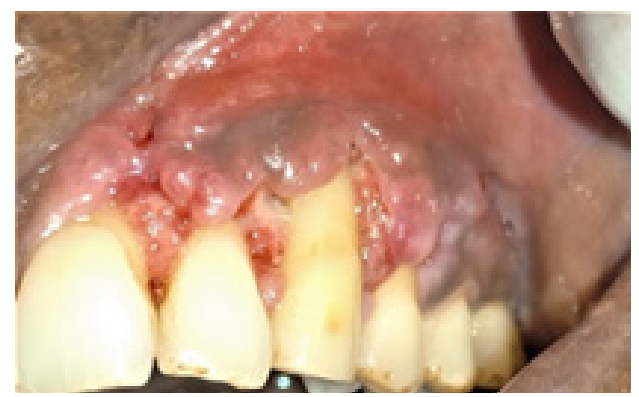

Figure 11: 1-week Post-operative Healing

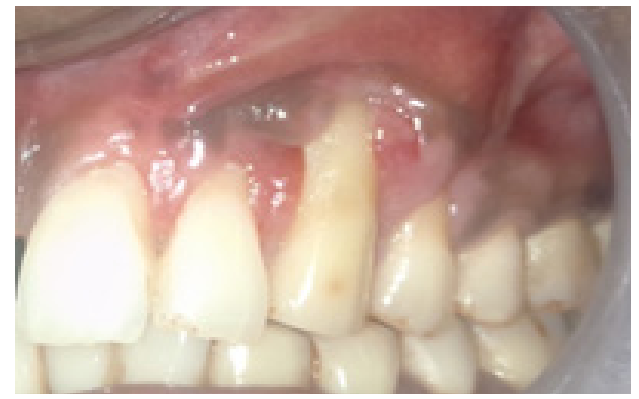

Figure 12: 1-month Post-operative Healing

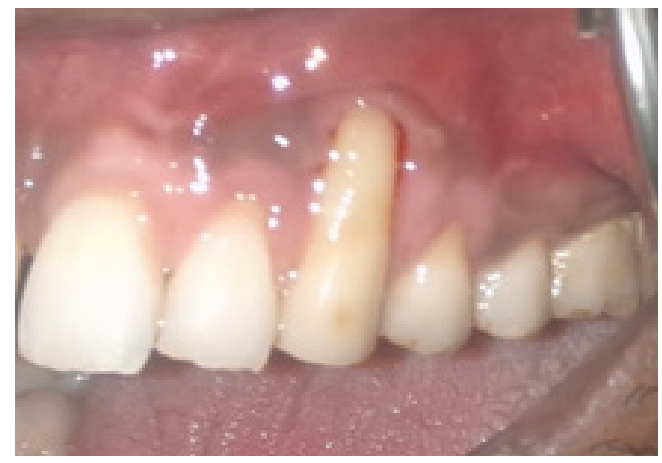

Figure 13: 6 Months Post-operative Healing

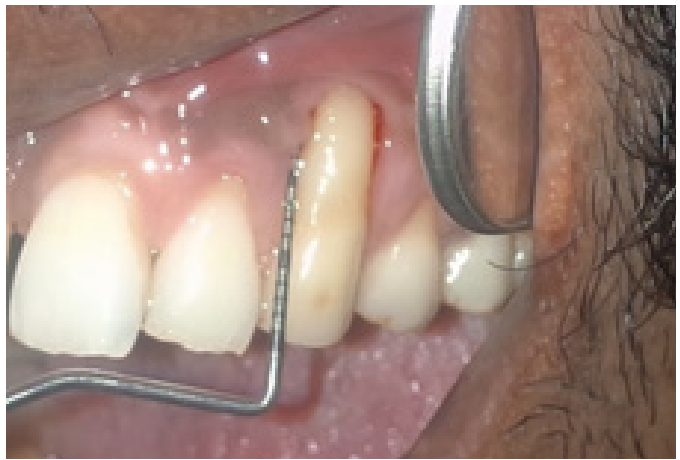

Figure 14: Reduction of Probing depth to $3 \mathrm{~mm}$

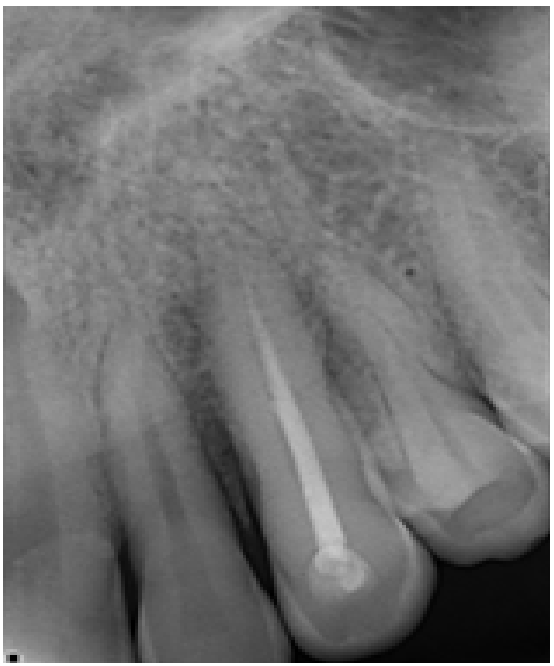

Figure 15: 6 Months postoperative radiograph showing bone fill in surgical site

\section{Discussion}

The successful clinical outcome for the treatment of an Endo-Perio lesion lies in proper diagnosis, treatment plan and regeneration of the lost tissue structures. The amniotic membrane acts as a reservoir of Multi-Potent Stem cells which aid in the acceleration of the healing process along with which it has many advantages over the other materials in properties such as:

- Immune modulatory effects

- Anti -scaring and anti-inflammatory properties

- $\quad$ Reduced pain

The use of amniotic membrane along with Bone graft increases the osteoinductive and osteoconductive properties thereby enhancing the formation of the alveolar bone. When compared to traditional GTR membranes, placental barriers, Amniotic membrane demonstrate many unique properties. First and foremost, the Amniotic membrane possesses a variety of proteins that provide a bioactive matrix to facilitate wound healing, including collagen types I, III, IV, V, and VI, laminin. In addition to providing a bioactive matrix, studies have shown 
placental barriers to have antibacterial properties and to reduce inflammation via inhibition of macrophages and polymorphonuclear neutrophils. Amniotic membrane is extremely thin, averaging $50 \mu \mathrm{m}$ thickness. Unlike many barrier membranes, the Amniotic membrane is self-adhering and does not require sutures for fixation. The amniotic membrane does not require precise trimming for adaptation. The minimal thickness of this barrier membrane, combined with its self-adherent nature, allows it to intimately adapt to contours around roots and over defects and does not require chair-side or in situ fabrication. In the present case, a scenario the patient's chief complaint was swelling and pus discharge which on examination revealed to be a non-vital tooth and the radiographic findings suggested extensive bone loss around the mesial and distal aspects of 23. The tooth also exhibited a grade III mobility for which the initial treatment plan was extraction of the tooth. Since the patient was not willing for extraction and concerned more about retaining the natural tooth's form and function alternate treatment plans were made. After initial Phase I therapy and root canal therapy the Grade III mobility has reduced to Grade I. hence occlusal reduction was done instead of splinting. At three months post operatively the mobility has completely reduced and the tooth has returned to form and function. The reduction in pocket depth and gain in the clinical attachment level is suggestive of new attachment or re-attachment and regeneration of periodontal tissues. As stated by Mishra et al. (2014), the amniotic membrane firmly adheres with the wound through fibrin and elastin linkages that seals the wound and prevents contamination. The lymphatic integrity is restored and circulating phagocytes are protected from exposure. The tight adherence allows faster removal of surface debris and bacteria from the wound. Cystatin $\mathrm{E}$ which is an analog of cysteine proteinase inhibitors is responsible for its antiviral properties (Chopra A. et al., 2013). The exposed nerve endings are protected from the external irritant by the soft mucoid lining that helps to decrease pain sensation. Increased extracellular matrix deposition MSCs differentiation helps in regenerating the damaged tissue and regulates the local cellular responses to injury by paracrine signaling. The amniotic membrane provides a better state of hydration that soothes the wounded and diminishes inflammation thereby reducing pain. The anti-scarring property is due to the proper maintenance of balance between TGF-1 and TGF-3 due to the secretion of Vascular Endothelial Growth Factor and Hepatocyte growth factor by the amniotic membrane. The amniotic membrane closely mimics the basement membrane of the human oral mucosa. It forms the acellular dermal matrix which is a cellular biocompatible human connective tissue matrix. After 6 months postoperatively, the tooth became functional and stable. 


\section{References}

1. Bhushan KS, Singh G, Chauhan G, Prakash S (2015) Amniotic membrane \& its structure, features and uses in dentistry-a brief review.Int J Sci Adv Res Technol 3: 354-360.

2. Chakraborthy S, Sambashivaiah S, Kulal R, Bilchodmath S (2015) Amnion and chorion allografts in combination with coronally advanced flap in the treatment of gingival recession: A Clinical Study. J Clin Diagn Res 9: ZC98.

3. Shah R, Sowmya NK, Mehta DS (2014) Amnion membrane for coverage of gingival recession: A novel application. Contemp Clin Dent 5: 293.

4. Srivastava R, Siddiqui ZR, Jhingran R, Bains VK (2016) Double Papilla Graft with Amnion Membrane for Root Coverage of Isolated Recession. World J Dent 7: 213-216.

5. Chaitanya Pradeep Joshi, Alisha Altaf Panjwani, Cynthia Bernardo D'Lima, Nitin HemchandraDani (2017) Comparative Evaluation of Amnion-Chorion Membrane and Chorion Membrane for Root Coverage and Gingival Biotype Enhancement: A Case Report.EC Dent Sci 255-259.

6. Mahajan R, Khinda PK, Shewale A, Saravanan SP (2015) Guided Tissue Regeneration Based Treatment of Root Coverage using Placental Membrane Allograft: A Case Report. Int J Sci StudyCase Reports \& Reviews 2: 1-5.

7. Kothiwale SV, Anuroopa P, Gajiwala AL (2009) Clinical and radiological evaluation of DFDBA with amniotic membrane versus bovine-derived xenograft with the amniotic membrane in human periodontal grade II furcation defects. Cell Tissue Bank 10: 317 .

8. Holtzclaw, Dan \& J. Toscano, Nicholas (2013) AmnionChorion Allograft Barrier Used for Guided Tissue Regeneration Treatment of Periodontal Intrabony Defects: A Retrospective Observational Report. Clin Adv Periodontics 3: 131-137.

9. Al-Fouzan KS (2014) A new classification of endodontic-periodontal lesions. International journal of dentistry.

Submit your manuscript to a JScholar journal and benefit from:

ฯ Convenient online submission

ฯ Rigorous peer review

ๆ Immediate publication on acceptance

q Open access: articles freely available online

q High visibility within the field

ब Better discount for your subsequent articles

Submit your manuscript at http://www.jscholaronline.org/submit-manuscript.php 